\title{
A PROGRAM TO CHANGE MANAGEMENT OF DAIRY FARMS TO REDUCE JOHNE'S DISEASE PREVALENCE
}

\author{
Wapenaar, W. ${ }^{1}$, Leids, $P^{3}$, Denney $L^{3}{ }^{3}$, Van Schaik, G. ${ }^{2}$, Stehman,.$^{2}$, Schukken, Y.H. ${ }^{2}$, Huntley, $J^{3}$ \\ ${ }^{1}$ Veterinary Faculty, Utrecht University, Utrecht, The Netherlands, e-mail: wwapenaar@planet.nl \\ ${ }^{2}$ Department of Population Medicine and Diagnostic Sciences, Cornell University, Ithaca, NY, USA. \\ ${ }^{3}$ Division of Animal Industry, Department of Agriculture and Markets, New York State Government
}

New York State cattle health insurance program (NYSCHAP) was developed in New York State to address concerns with regard to quality assurance and biosecurity in New York State cattle herds. The goal of the program is to reduce disease prevalence by changing the management practices and increase the biosecurity of a farm. State veterinarians who visit the farms annually carry out the program. During visits a risk analysis of the farm is carried out and management changes are evaluated. Furthermore, farms can test for diseases against a reduced rate. The overall objective of the present study was to evaluate the Johne's disease module of NYSCHAP. Firstly, a global overview of the farms that are engaged in the NYSCHAP was made. Secondly, management changes on a subgroup of farms were investigated. Results will show the potential of the NYSCHAP to improve biosecurity on dairy farms.

The baseline data consisted of 20 variables that were collected from the 426 NYSCHAP participants. The data used for the management implementation study were collected from in total 50 reviews of 30 farms. SPSS-databases were constructed to perform aggregated statistical analysis on these data. The average NYSCHAP-herd was larger than the average dairy herd in New York State, similarly herd production averages are above State average. Sixty-four percent of NYSCHAP-farms use a computer to record information. The goals for participation in the program that were mentioned most frequently were: 'limit transmission of the disease', 'become a Johne's negative herd' and 'gain information and have knowledge to change management on the farm'. Most of the farms (94\%) intended to do some kind of herd testing for Johne's disease and $83 \%$ have the intention of testing the whole herd. Seventy-one percent mentioned Kinetics ELISA combined with fecal culture as their intended test approach. The average number of biosecurity measures advised to change on a farm was 17 . The average percentage of management practices that were actually implemented as evaluated by reviews in 1999 was $79 \%$, which means that the average farm implemented 13.4 of the advised measures. The most implemented measures were: cull (suspected) clinical cases, avoid manure contamination, have a designated clean and dry calving area and feed calves milkreplacer. The measures that were most often not-implemented were: one animal in each calving pen, identify likely positive animals, segregate age groups and perform testing on purchased animals.

The detailed data as collected in NYSCHAP, result in a good impression of the farm and management on the farm. Johne's disease can only be successfully eliminated from a farm when proper management is in place. Therefore, being able to change management at the farm is an important achievement. NYSCHAP was shown to be successful in changing health management on farms. 